\title{
Sénégal : la santé au risque de l'information
}

Senegal : health risk information

Senegal : informazioni sui rischi di salute

\section{Seydou Nourou Sall}

\section{CpenEdition}

\section{Journals}

Édition électronique

URL : http://journals.openedition.org/ctd/1016

DOI : $10.4000 /$ ctd. 1016

ISSN : 2491-1437

Éditeur

Chaire Unesco Pratiques émergentes en technologies et communication pour le développement

Édition imprimée

Date de publication : 30 octobre 2016

ISBN : 2491-1437

Référence électronique

Seydou Nourou Sall, «Sénégal : la santé au risque de l'information », Communication, technologies et développement [En ligne], 3 | 2016, mis en ligne le 30 octobre 2016, consulté le 12 juin 2020. URL http://journals.openedition.org/ctd/1016 ; DOI : https://doi.org/10.4000/ctd.1016

Ce document a été généré automatiquement le 12 juin 2020.

Communication, technologies et développement 


\title{
Sénégal : la santé au risque de l'information
}

\author{
Senegal : health risk information \\ Senegal : informazioni sui rischi di salute
}

Seydou Nourou Sall

\section{Introduction}

2 Le recours aux pratiques mystiques, à la voyance et/ou divination, à la médecine traditionnelle est devenu tellement manifeste et a pris une telle proportion dans la société sénégalaise qu'il n'est pas exagéré de nourrir quelques inquiétudes. Preuve par la revue de presse. Les journaux sénégalais ont, dans une sorte de chorale, rapporté les fréquentes profanations de tombes au cimetière de Pikine, dans la banlieue dakaroise. Le Quotidien ${ }^{1}$ rend ainsi compte de cette insolite découverte :

Il y a deux mois, deux corps de nouveaux nés ont été jetés au milieu des tombes. Quatre mois avant, une dame a été déterrée au cimetière de Pikine. (...) Dans la nuit du jeudi au vendredi, deux corps, celui d'un vieux âgé de 80 ans et d'un bébé de 7 mois, ont été extraits de leur tombe. (...). Concentrés sur cette affaire, les policiers ne sont pas au bout des découvertes macabres: Ils retrouvent, à côté des tombes profanées, une tête de chèvre enveloppée dans un linceul.

Selon la presse et les premiers éléments de l'enquête, les profanateurs auraient été guidés par « des pratiques mystiques».

Dans le champ politique, la bataille mystique a semblé prendre le pas sur la force des arguments dans les stratégies de conquête et d'exercice du pouvoir. Ce sont deux candidats à la Présidence de la République qui, plusieurs mois après leur élection, refusent d'emménager au Palais - la résidence présidentielle - tant qu'il n'a pas été " déminé $»^{2}$. C'est un Président de la République, candidat à sa propre succession, qui accuse un ex-partisan de sacrifice d'êtres humains ${ }^{3}$. C'est du sang d'origine inconnue que l'on déverse devant le siège d'un candidat à l'élection présidentielle. C'est un 
ministre qui enterre des bœufs vivants dans un cimetière musulman pour échapper aux audits. Ce sont tous les soupçons qui pèsent sur la mystérieuse disparition d'enfants durant la campagne électorale de l'élection présidentielle de février 2012. Dans le domaine médical, un responsable de l'association sénégalaise des personnes diabétiques, invité du journal de 12 heures de RFM du 21 novembre 2015, sonnait ainsi l'alerte : « beaucoup de diabétiques se dirigent vers la médecine traditionnelle, pensant soulager leurs maux. Ils ne consultent les vrais spécialistes qu'en cas de situation désespérée ».

5 C'estdire que les pratiques mystiques et celles liées à la médecine traditionnelle quiétaientautrefois dissimulées par nombre de Sénégalais sont aujourd'hui décomplexées. Les charlatans, voyants, guérisseurs, etc., occupent de plus en plus l'espace et se posent comme acteurs incontournables de la santé mentale et physique de leurs compatriotes au détriment ou contre, c'est selon, la médecine moderne. Toutes les vieilles superstitions traquées par les religions du livre amorcent un retour triomphal.

6 Ce subit regain d'intérêt des Sénégalais pour la voyance, les pratiques mystiques et la médecine traditionnelle coïncide comme par hasard avec la surmédiatisation de ces dits phénomènes et de leurs acteurs. En leur offrant cette visibilité, les médias ne donnent-ils pas de la légitimité à ces nouveaux acteurs qui semblent pouvoir trouver remède à tout ? Ou, en tant que simple reflet de la réalité, ne font-ils pas que surfer sur cet intérêt, ce crédit aveugle accordés à toutes ces formes de superstition?

\section{Des médias au Sénégal}

7 La recherche scientifique témoigne d'une abondante et riche documentation sur les médias au Sénégal. «Des débuts de la presse » (Pasquier, 1962) dans ce pays de l'Afrique de l'Ouest à " nos jours " (Barry, 2013), beaucoup de domaines ont été explorés. Les deux auteurs cités rendent compte du pluralisme médiatique sous la colonisation malgré la volonté manifeste de l'administration coloniale après la création du Moniteur Africain "de ne pas faire naître le besoin d'une autre feuille". Sous ce rapport, les premières années d'indépendance peuventêtre considérées comme une période de régression. Léopold Sédar Senghor instaure un régime de parti unique qui ne pouvait qu'étouffer la libre expression de points de vue divergents. S'ouvre alors une ère de contrôle politique des médias (Paye, 1992 ; Tudesq, 1995). Cette période sombre de la presse sénégalaise prend fin avec l'accession d'Abdou Diouf à la magistrature suprême qui libéralise tous azimuts. Des périodiques comme Sud Hebdo, Walf Hebdo, Le Cafard Libéré, Le Témoin vont se développer à la fois indépendamment des organisations partisanes et en offrant une alternative à la voix officielle de l'organe gouvernemental, Le Soleil (Touré, 1991). Cette évolution de la presse privée au Sénégal a permis de rompre avec

8 « le journalisme de communiqués, offrant un écho des déclarations officielles, reflétant les préoccupations de la classe des notables, mais négligeant les attentes et les besoins des masses» (Frère, 2000, p. 41). Cette libéralisation s'accompagne d'un souci de structuration avec la création d'associations et d'organisations professionnelles reconnues par les pouvoirs publics (Paye, Op. cit.). Le Syndicat des professionnels de l'information et de la communication du Sénégal (SYNPICS), né en 1984 sur les cendres de l'Association nationale des journalistes sénégalais (ANJS), fait preuve de plus de 
dynamisme dans la défense des droits des journalistes. Un organe d'autorégulation est créé en 1999 : le Conseil pour le respect de l'éthique et de la déontologie (CRED). L'État avait auparavant mis sur pied un organe de régulation de la Radio-télévision (HCRT), remplacé en 1998 par le Haut Conseil de l'audiovisuel (HCA), devenu par la suite le Conseil national de régulation de l'audiovisuel (CNRA).

Ce pluralisme médiatique doublé d'une relative indépendance, tant sur le plan financier que politique, a été déterminant dans la défaite électorale d'Abdou Diouf en mars 2000 (Ndiaye, 2002). L'accession du libéral Abdoulaye Wade à la Présidence de la République va consolider et renforcer la libéralisation et le multipartisme, toutefois sur fond de rapports heurtés entre presse et pouvoir (Havard, 2004). Mais tout compte fait, son moment de gloire, la presse sénégalaise l'avait enfin vécu lors de la présidentielle de 2000 et pouvait, en tant que telle, être sacrée contre-pouvoir.

Les médias sénégalais ne peuvent, cependant, continuer à jouer pleinement ce rôle de contre- pouvoir qu'en étant libres et indépendants. Or, pour plusieurs raisons, cette indépendance est à relativiser. L'action de certains groupes de pression pousse Ndiaga Loum à décréter

11 «l'impossible autonomie » des médias sénégalais (Loum, 2003). Comment, par ailleurs, des médias qui dépendent manifestement des subventions de l'État pour assurer leur pérennité peuvent-ils avoir la prétention d'être libres? En effet, « depuis 1996, l'État accorde une aide à la presse en mobilisant des fonds relativement importants... Une aide sans impact ni sur les entreprises de presse pour les rendre performantes ni sur les conditions de travail, encore moins sur les conditions sociales des journalistes » (Faye, 2015). Pire, Mor Faye explique que ce fonds est devenu un instrument dont se servent les pouvoirs en place pour exercer une pression sur les médias. Pour relativiser l'autonomie de ces derniers, il faut enfin relever ce phénomène peu reluisant et négateur de la liberté de presse : la corruption d'une bonne partie de la presse privée par des hommes politiques et hommes d'affaires (Faye, 2008).

Le seul facteur sur lequel n'ontpas insisté ces auteurs est l'impact du public dans la construction del'information, unedictaturedel'audimatquiconstitueunvéritablefreinàl'indépendancedes médias. Cette étude se propose d'explorer cette piste en tentant d'expliquer la surmédiatisation des phénomènes liés à la voyance, à la divination et à la médecine traditionnelle par le souci des médias de coller aux attentes de leur audience.

\section{Du rôle des médias}

13 Avant d'aller plus loin dans l'analyse, un positionnement théorique s'impose. La presse est souvent interpellée dans les situations de conflits, de crise, de risques pour le développement, etc. Cela voudrait-il dire que les journalistes ont un rôle de prévention à jouer ou est-ce tout simplement une manière de faire jouer leur sens de la responsabilité?

14 L'information - qui est la première fonction des médias - est en principe un champ illimité et recouvre les fonctions éducatives et sociales. Mais la course à l'audimat a fortement restreint ce volet essentiel. Le journalisme se résumant, la plupart du temps et de plus en plus, à la description du superficiel, du pittoresque, de l'accidentel. La presse parle plus volontiers des «trains qui arrivent en retard que des trains qui 
arrivent à l'heure", des conflits que de l'activité normale des organisations. Par ailleurs, des interrogations se sont posées et continuent d'agiter le milieu de la presse : le journaliste, dans le traitement de l'information doit-il avoir comme souci de poursuivre des objectifs spécifiques? Cette façon d'orienter le travail des professionnels de l'information ne constitue-il pas une négation de la liberté de presse ? La réflexion développée à partir des années 1970, notamment dans le monde anglo-saxon, autour du rôle des médias dans les situations de crise, est toujours d'actualité. Le courant "Médias et construction de la paix », qui prône la nécessité d'orienter le travail des journalistes vers la recherche et la consolidation de la paix, rencontre aujourd'hui encore des détracteurs. Ces derniers pensent qu'obliger la presse à travailler pour servir des objectifs spécifiques est une entorse à la liberté de presse (Frère, dir., 2005).

15 A vrai dire, la grande difficulté qu'a le personnel des médias à s'ériger en "développeur» est liée au fait que le mot «médias de développement » est chargé négativement. C'est le rôle que les pères de l'indépendance ont voulu faire jouer aux médias qui naissaient sur les ruines de la presse coloniale. Ils étaient utilisés presque partout "pour des programmes d'alphabétisation des masses, et comme support supplémentaire ou classes à distance pour les programmes des écoles primaires et secondaires [...] pour promouvoir la santé publique, comme les campagnes d'immunisation et la lutte contre les problèmes de santé endémiques... " (Karikari, 2007,

16 p. 16). En réalité, les fonctions éducatives (Dia, 1987) et sociales n'avaient d'autre signification que d'assigner aux médias un rôle de propagande, de courroie de transmission entre le pouvoir et le peuple, de légitimation du régime (Tudesq, 1995, p. 81). Une position idéologique qui a servi de prétexte pour les dirigeants africains des premières années d'indépendance de supprimer la liberté de presse et aux régimes en place d'exercer une sorte de tutelle sur les médias, de centraliser l'information. Dans leur entendement, « la critique ne pouvait être autorisée dans une nation en formation, pauvre de surcroît, où toute forme de journalisme n'allant pas dans le sens de la volonté gouvernementale constituait un luxe, un gaspillage d'énergie et de ressources » (Frère, 2000, p. 31).

17 Même dans le cas où les dirigeants africains étaient animés de sentiments nobles, cette façon de concevoirlacommunicationauservicedudéveloppementétaituneentrepriseperdued'avance. Il a été démontré que le modèle vertical, up down, diffusionniste, de la communication pour le développement n'était pas la meilleure méthode de faire adhérer les populations à des causes, résoudre des conflits, changer leurs comportements de manière durable. «En effet, dans de nombreux cas, les populations ont développé des comportements de résistance plus ou moins directs face à des interventions qui leur «tombaient dessus» sans que leurs préoccupations, leurs besoins, leur vision, leur participation et leur point de vue aient été sollicités d'une part, sans que les motivations des acteurs du développement leur aient été clarifiées au préalable d'autre part» (FAO, 2002). C'est l'expérience qui a donc fini par démontrer qu'il fallait désormais privilégier le paradigme de la communication down-up et participative plus efficace dans les interventions de développement et de modernisation (Bessette, 2004). 
D'où l'intérêt accordé de nos jours aux radios communautaires par les organismes internationaux. Car, dans ce modèle de communication pour le développement, ces médias du tiers-secteur jouent un rôle important :

Les radios indépendantes contribuent à la démocratisation de la communication et à la participation active de la population. Celles-ci donnent des outils nécessaires à la communauté dans l'analyse des problèmes et la recherche de solutions. Elles permettent l'accès à l'information, donnent une « voix » à des populations souvent marginalisées et fomentent l'exercice démocratique tout en valorisant les cultures locales. (Tedesco, 2007-2008).

19 Pour autant, et nonobstant les autres raisons évoquées ci-dessus, les médias traditionnels doivent-ils laisser ce terrain auxmédiasdeproximité? Lesprofessionnelsdesmédiasnepeuvent et/ou doivent-ils pas, sans se départir de leur professionnalisme, jouer un rôle dans le domaine de l'éducation, de la santé, de l'agriculture, de l'environnement, du développement durable etc. ? S'ils ont du mal, ne doivent-ils pas se garder, au moins, de desservir le développement?

\section{Du monde de l'imaginaire et de la médecine traditionnelle}

Dans cette étude, il est surtout question du monde de l'imaginaire et de la médecine traditionnelle qui ont, en Afrique, quelque chose à voir avec le religieux. La religion y est «l'armature de la vie», "charpente toutes les actions publiques et privées de l'homme " (Dieterlen, 1965, p. 9). Bien que les religions importées - christianisme et islam - y soient durablement installées, "comprendre l'Afrique et l'Africain sans l'apport des religions traditionnelles serait ouvrir une gigantesque armoire vidée de ses contenus les plus précieux" (Dieterlin, ibid.). Et ce mode de croyances et de représentations valorise plus que les autres la dimension guérison; il est le seul à accorder de l'importance à la voyance et à la divination. Les devins, guérisseurs, prêtres, voyants sont sollicités pour tous les aspects de la vie, par le jeune marié qui veut un enfant, le cultivateur pour une bonne récolte, le pêcheur pour une bonne prise, le malade pour sa guérison, le commerçant pour attirer la clientèle, etc. Ils sont les personnages centraux (Damome et Delcroix, 20015) de ce système cosmogonique, de ce monde parfaitement organisé.

La conscience claire qu'ont les Africains de ce qui les entoure les a poussés à analyser, dans les moindres détails, tous les éléments de l'univers qu'ils ont appréhendés. Ceux-ci sont classés en catégories décomposables et mises en parallèle. [....] Ces catégories sont en correspondance les unes avec les autres, dans des relations non point seulement conceptuelles, mais biologiques, comme celles qui lient les organes d'un même corps vivant. (Dieterlin, Op. cit., p. 26).

Les amulettes, les masques, les gris-gris et danses rituelles et/ou initiatiques forment le décor de cet univers qui faisait la richesse de l'Afrique traditionnelle.

Ces pratiques et formes traditionnelles de représentations qui procuraient aux peuples d'Afrique leur force spécifique et leur originalité ont, par la suite, souffert, en ce qui concerne le Sénégal, du dur combat que leur ont mené aussi bien les religions importées que les pouvoirs politiques. Ces religions se sont posées en s'opposant radicalement et violemment aux cultes et pratiques traditionnels, dits "païens » et « idolâtres ». Quant aux autorités étatiques, elles ont pendant longtemps considéré le 
maintien des religions traditionnelles comme constituant un danger pour la survie de la nation.

«Étant donné que, malgré de nombreux traits communs, les religions traditionnelles diffèrent grandement suivant les ethnies - on aurait dit autrefois que chaque tribu avait sa religion propre -, les autorités craignent que ces religions contribuent à maintenir le " tribalisme » et entravent donc l'intégration nationale. Les autorités n'encouragent pas la pratique de ces religions, en dépit des efforts officiels allant dans le sens d'un "retour à l'authenticité » " (Hesseling, 1985, pp. 86-87).

Elles toléraient seulement quelques-unes de leurs manifestations extérieures telles que masques, danses et rites, pour des motifs d'ordre touristique et au nom du folklore national.

Toutefois, malgré ces contraintes, ces modes de représentations arrivent toujours à s'exprimer. La croyance en des génies tutélaires - Mame Coumba Mbang à Saint-Louis, Coumba Castel à Gorée, Mbossé à Kaolack, Ndëk Dawur à Dakar, Mam Koumba Lambay à Rufisque - (Monteil, 1980, p. 66), aux vertus protectrices des amulettes et danses rituelles, reste toujours d'actualité au Sénégal. Ainsi voit-on souvent « dans l'islam noir, tel qu'il se vit aujourd'hui, une forme plus ou moins bâtarde de la tradition orthodoxe, une religion de compromis dont aucun vrai musulman ne saurait s'accommoder, mais sur laquelle en l'occurrence, il est opportun de fermer les yeux » (Thomas et Luneau, 1995, p. 313).

Quant à la médecine traditionnelle, elle n'a jamais totalement disparu du décor africain et la tendance est plutôt à sa réhabilitation.

La place du guérisseur traditionnel est, encore très importante. Sa présence est rassurante dès que l'on reconnait son pouvoir de dialoguer avec les forces obscures. Il porte, en effet, en lui, le pouvoir d'entrer en relation avec les forces du Bien et du Mal, de les mater ou d'en déclencher les mécanismes terrifiants. Il appartient, dans l'imaginaire social, à une élite détentrice d'un savoir, la connaissance de l'occulte. (Damome et Delcroix, art. cité, p. 56).

Il serait sage, pensent ces auteurs et certains autres experts, d'introduire la médecine traditionnelle et ses thérapies à base de plantes dans les systèmes officiels de santé publique. D'autantplus, disent-ils, qu'en 2000, le Comité régional africain de l'Organisation mondiale de la santé a recommandé « un développement accéléré de la production locale de médicaments traditionnels" dans sa résolution intitulée Promouvoir le rôle de la médecine traditionnelle dans les systèmes de santé: Stratégie pour la région africaine (Damome et Delcroix, ibidem).

Ces recommandations semblent être prises en compte par le Sénégal qui, à travers la création d'une Direction de la médecine traditionnelle rattachée au Ministère de la santé, officialise de fait l'introduction de la médecine traditionnelle dans son système de santé publique bien que la loi réglementant le domaine ne soit pas encore promulguée.

$29 \mathrm{Au}$ vu de ce qui précède, force est de constater qu'il n'est pas, ici, question de discréditer le traditionalisme. La vérité est que tout ce qui vient de la tradition n'est pas mauvais, tout ce qui relève de la modernité n'est pas bon non plus. Ce qu'il faut, c'est arriver à différencier la vraie médecine traditionnelle du charlatanisme. La dernière catégorie regroupe tous ces guérisseurs, voyants et/ou devins qui inondent le quotidien des Sénégalais, relayés par les radios et télévisions, la presse écrite et sites internet. Ces charlatans, en véritables vendeurs d'illusions ou «faiseurs de miracles », exposent les 
Sénégalais à de graves problèmes de santé publique avec la complicité tacite de la presse, leur porte d'entrée. Il aurait été, pourtant, intéressant pour les médias de vulgariser les recherches et applications intéressantes sur la pharmacopée traditionnelle développées à la Faculté de médecine de l'Université Cheikh Anta Diop de Dakar ou au Centre Malango de Fatick. Au lieu de cela, ces questions ne sont analysées que sous l'angle du sensationnel, de l'extraordinaire, au risque de desservir la santé.

\section{Méthodologie}

Les avis trimestriels du Conseil national de régulation de l'audiovisuel (CNRA) vont servir de base d'analyse à cette étude qui se veut qualitative. Le CNRA est l'organe de surveillance des médias sénégalais. Sa mission est de veiller à la liberté de l'information et de la communication, à la libre et saine concurrence entre les entreprises de communication audiovisuelle, de concilier l'acte de communiquer sans entrave et le respect des règles devant l'accompagner. En cas de manquement, il peut s'autosaisir ou être saisi par les consommateurs; des sanctions sont alors prévues en cas de violation de la loi. D'après l'article 26 portant création du CNRA, elles peuvent être de plusieurs ordres: suspension totale ou partielle d'un programme, sanctions pécuniaires, fermeture de l'organe de presse et retrait de la fréquence. En guise d'avertissement et pour faire preuve de pédagogie, l'organe de régulation publie des avis trimestriels qui relèvent les dysfonctionnements et des manquements dans les programmes de certains médias audiovisuels et formule des recommandations. Les émissions consacrées à la voyance et/ou divination, la "publicité mensongère " liée au charlatanisme y sont régulièrement épinglées.

31 Cequ'enpensentlesorganisationsprofessionnelles- OrdredesmédecinsduSénégal, Fédération sénégalaise des praticiens de la médecine traditionnelle (Fsmt) reconnue officiellement, Syndicat des professionnels de l'information - est également important pour l'analyse. Leurs sorties sont exploitées dans ce travail. De pertinents dossiers sur ces phénomènes ont été également réalisés par la presse elle-même. Ils permettent de comprendre les raisons de la surmédiatisation. Pour affiner, nous aurions voulu disposer de statistiques fiables sur la place de ces émissions dans l'audience des médias. Cette volonté s'est heurtée, malheureusement, à l'irrégularité des mesures d'audience au Sénégal. Et lorsqu'elles existent, elles se focalisent plus sur l'audience générale que sur celle de chaque émission prise individuellement.

\section{Les médias au service de la santé ?}

Le législateur sénégalais a très tôt compris la sensibilité des questions liées à la santé et leur exploitation par les moyens de communication de masse. C'est ainsi que l'article 14 de la loi $\mathrm{N}^{\circ}$ 83-20 du 28 janvier 1983 relative à la publicité précise : « La publicité ne doit pas éveiller chez les malades des espoirs fallacieux, ni exploiter leur manque éventuel d'esprit critique

l'égarddesmessagesleurpermettantuntraitementefficaceoulaguérison ".

Ordesmessagesdu genre «Aladji serigne cissé, Grand marabout mandingue, Voyanceguérisseur-tradipraticien, vous assiste sur tous vos problèmes, Santé, travail, amour, voyage, concours $(s i c)^{4}$ » sont très fréquents dans les petites annonces des quotidiens et 
journaux en ligne. Message toujours complété par une longue liste de maladies sensées pouvoir être traitées. Preuve par cette autre petite annonce :

Dr El Hadj Ona Ara, grand guérisseur en médecine traditionnelle. Maladies à traiter : hémorroïde intérieur et extérieur (sic), dermatos (re-sic), ballonnement, mauvaise haleine, faiblesse sexuelle, éjaculation précoce, infection, prostate, hépatites, asthme, hernie, ulcère, fibrome, stérilité, sinusite, maux de reins, pipi au lit, femme de nuit, mari de nuit, aide-mémoire, orientation de travail, protection, quelqu'un qui a un problème avec son travail ou société, problème de couple. Par la grâce de Dieu tous vos problèmes seront résolus 5 .

Ils peuvent d'ailleurs se passer de publicité parce que les professionnels des médias se chargent de faire leur promotion dans des émissions consacrées à la santé. C'est ainsi qu'invité de l'émission Wer gu yaraam ${ }^{6}$ de Sud FM Saint-Louis du 15 novembre 2015, un tradi-praticien explique qu'il peut soigner l'hémorroïde, l'impuissance sexuelle, la stérilité, la cataracte, la tension des yeux, etc. devant une animatrice tellement convaincue qu'elle passait ses coordonnées téléphoniques et son adresse en direct.

La télévision exploite, elle aussi, massivement ce créneau avec des émissions exclusivement dédiées. La SENTV, diffuse en prime time une émission ${ }^{7}$ qui met en vedette guérisseurs, féticheurs et toutes sortes d'adeptes de pratiques mysticoreligieuses. L'animateur fait un zoom hebdomadaire sur un spécialiste de ces questions qui, exemples à l'appui, et avec tout l'arsenal mystico-ésotérique, explique comment il soigne un patient atteint de mauvais sorts ou malmené par des esprits maléfiques. Toujours sur la même chaîne, l'émission Wer Gui Yaram, met en vedette un soit disant phytothérapeute qui donne des conseils de santé par les plantes. Son audace a d'ailleurs attiré l'ire de l'ordre des médecins du Sénégal qui a saisi la Division des Investigations criminelles d'une plainte pour exercice illégal de la profession ${ }^{8}$. Le président de l'Ordre a expliqué aux enquêteurs que ce tradi-praticien a établi des bulletins d'analyse à l'ordre d'une femme, en date du 25 octobre 2013. Dans ces bulletins, après avoir diagnostiqué une " hypertension », le phytothérapeute a demandé des examens portant sur une "écographie abdominale" ainsi que d'autres analyses médicales. Pour le président de l'Ordre, « ces faits sont suffisamment graves, parce qu'ils troublent l'ordre public en mettant en péril la santé des citoyens, surtout quand cela s'accompagne d'une publicité à la SenTv (.)

Il viole la loi No 66-69 du 04 juillet 1996, relative à l'exercice de la médecine et à l'Ordre national des médecins, ainsi que le décret n 67-147 du 10 février 1967, instituant le Code de déontologie médical ».

36 Au début épiphénomène de presse écrite, le charlatanisme s'est maintenant généralisé à tous les supports. Il n'y a plus une radio ou une télévision qui ne lui réserve pas une place dans la grille des programmes. Presque toutes disposent également de séances de consultation de spécialistes de la magie, de la divination ou de la voyance au cours desquelles des sorts sont décryptés et des conseils prodigués dans tous les domaines: activités professionnelles, vie conjugale, difficultés sanitaires, projets de voyage ou d'étude. Sentant le filon, une fréquence radio, SAPHIR FM, s'est spécialisée dans la voyance en direct et y tire l'essentiel de ses recettes. Dans un dossier sur la voyance, la Gazette explique que le serveur vocal installé à cet effet et chèrement taxé assure la santé financière de la radio, générant un millions de francs CFA de recettes journalières. des médias, au coin d'une ruelle, au marché, crèvent aujourd'hui l'écran, occupent de 
plus en plus l'espace et fonctionnent comme soupape de sécurité pour une population en détresse. Ils exploitent la misère matérielle et/ou morale de la masse en lui garantissant des solutions à tous les maux. En leur offrant cette visibilité, les médias font ainsi la publicité et donnent de la légitimité à ces nouveaux acteurs. Or un simple respect des règles d'éthique et de déontologie oudestextesrégissantlesmédiasauraitdûrecommanderlanonmédiatisationdecespratiques.

Les organes de régulation et autres organisations professionnelles seraient-ils impuissants? Tous ces produits médiatiques ont été épinglés dans les avis trimestriels du CNRA. Rien que pour l'année 2014, trois ont porté sur ce phénomène. L'avis trimestriel du 17 janvier 2014 fustige "la diffusion d'émissions de voyance en direct avec appels téléphoniques sur serveurs et d'autres servant de tribune à des guérisseurs traditionnels ou des marabouts qui prétendent pouvoir soigner diverses maladies ». En guise de recommandations, le CNRA demande aux médias « de se garder de servir de tribune à des individus dépourvus de toute qualification légale et dont la promotion expose des populations non averties à des risques de détérioration de leur santé, soumet les professionnels de la santé à une concurrence déloyale, crée un environnement favorable à l'exploitation de franges importantes de la population qui accordent un certain crédit aux propos de ces individus relayés par les médias audiovisuels $»^{9}$. L'avis de janvier-février-mars 2014 est de la même veine. Les dysfonctionnements relevés sont liés d'une part aux «émissions de voyance, avec l'utilisation de serveur surtaxé sans indication de coûts à l'écran, au cours desquelles les animateurs prétendent répondre aux questions de toute nature du public " et d'autre part aux "émissions à caractère médical qui traitent de thématiques et de concepts par des tradi-praticiens autoproclamés dont la manipulation relève de professionnels et dont le caractère sensible préfigure des dangers pour la santé des populations $»^{10}$. L'avis de juillet-août-septembre 2014 ne dit pas autre chose. Il épingle, comme ses précédents, des dérives liées à "la diffusion d'émissions interactives de voyance sur une longue durée par la télévision LCS et, ceci, sans indication des coûts des appels sur serveur et au détriment de l'orientation éducative, informationnelle ou de divertissement que doit poursuivre les télévisions ${ }^{11}$. Quant à la Fédération des praticiens de la médecine traditionnelle, elle se désole que l'image de la médecine traditionnelle soit ainsi ternie par

«cette forme d'exposition interdite par la loi et dont les conséquences peuvent être graves pour les populations ». La presse, selon elle, n'accompagne pas la médecine traditionnelle.

40 «Car à travers les tapages et publicités mensongères diffusés à longueur de journée, [elle] est en train d'exposer les Sénégalais à de graves problèmes de santé publique $»^{12}$. Le Syndicat des professionnels de l'information et de la communication du Sénégal (Synpics) a aussi réagi pour dénoncer la surmédiatisation des voyants. Comment expliquer alors la persistance de telles pratiques? Ces organisations, le CNRA notamment, ne sont-elles pas outillées pour sévir ? La régulation serait-elle en panne au Sénégal?

42 La régulation des médias y souffre des nombreuses faiblesses du secteur médiatique en général : précarité matérielle, déficit de formation de nombre d'acteurs (notamment en journalisme et en gestion d'entreprise), manque de bonne volonté de certains patrons de presse, prolifération médiatique, corruption. L'irrespect des principes et outils 
régulateurs se comprend à la lumière de ces handicaps. S'il y a unanimité sur les termes et principes, les cas de violation de la loi, de l'éthique et de la déontologie, sont monnaie courante. Les organes de régulation sont d'autant plus impuissants qu'ils sont perpétuellement désapprouvés au sein même du microcosme médiatique. Il importe enfin de préciser qu'en théorie, l'organe de régulation est assez outillé pour faire face aux dérapages de toutes sortes, mais ses pouvoirs de sanctions sont fort limités. Ce qui explique la récurrence des problèmes qu'il dénonce depuis quelques années sans réelle capacité de coercition.

\section{Information ou publicité ?} n'ont en réalité rien de rédactionnel et sont, au contraire, tout ou partie promotionnelles. Elles se déroulent invariablement selon ce scénario. L'invité commence d'abord par raconter ses " prouesses médicales ». Il dresse ensuite une liste interminable de maladies qu'il peut soigner, raconte, en passant, qu'il a hérité cette science de ses parents et termine par donner ses coordonnées téléphoniques et son adresse de consultation. Un mélange des genres qui semble devenir la règle dans les médias sénégalais. Pourtant, l'article 11 de la loi relative à la publicité est limpide sur cette question :

La publicité doit pouvoir être clairement distinguée comme telle, quels que soient sa forme et le support utilisé. Lorsqu'elle est diffusée dans des médias qui comportent également des messages rédactionnels, la publicité doit être présentée de telle sorte que le consommateur puisse la distinguer facilement de ces messages.

En attendant que les médias veuillent bien, un jour se conformer à la réglementation, les acteurs de la voyance, de la divination et de la médecine traditionnelle se frottent les mains, tirent profit de ces émissions, sortes de publireportages ou de publicité tout court. Résultats :

Autrefois, les voyants réclamaient quelques pièces de monnaie pour intéresser les « djinns ». Cette époque révolue fait place à celle d'une marchandisation ardue de la pratique de la voyance. Entraînant la hausse considérable du prix de la consultation. Ce qui est devenu un marché subit ainsi l'implacable loi de l'offre et de la demande ${ }^{13}$.

L'hebdomadaire La Gazette explique que ce sont les devins les plus médiatisés qui dominent le marché. Il en est ainsi de Kor Mack Faye bombardé superstar de la divination par les médias :

Aux Hlm Grand Yoff, sa demeure attire une clientèle importante, particulièrement les célébrités de la lutte et de la musique. Chômeurs, femmes de ménage, cadres, commerçants, s'offrent les services de l'oracle. La séance de divination du voyantmarabout est rétribuée à $10000 \mathrm{~F} \mathrm{CFA}$. Un montant qui, du reste, n'est qu'un ticket d'entrée puisque les offrandes conseillées au client rendent souvent la note très salée ${ }^{14}$.

Sa consœur Selbé Ndom, elle aussi rendue célèbre par les médias, ne s'en tire pas mal :

La masse de fidèles agglutinée chaque jour devant sa porte, est une source importante d'argent. Chaque client débourse $10000 \mathrm{~F} \mathrm{CFA} \mathrm{pour} \mathrm{une} \mathrm{séance.} \mathrm{"} \mathrm{Il} \mathrm{y} \mathrm{a}$ au moins 20 personnes par jour ", informe un voisin. Cette moyenne rapportée au tarif unitaire de la consultation donne un montant de $200000 \mathrm{~F} \mathrm{CFA/jour,} \mathrm{soit} 6$ millions de F CFA par mois ${ }^{15}$. 

qu'encourager l'exploitation de la fragilité morale et matérielle de leur public ? Le fontils à dessein ? Et à quelles fins ? Ne savent-ils pas qu'à force de répéter les messages, ils pourraient inculquer des réflexes aux individus (Tchakhotine, 1952)? Kader Diop, l'ancien président du Comité pour le respect de l'éthique et de la déontologie (Cred, organe d'autorégulation) abonde dans ce sens quand il déclare que «ce type d'information devrait être tout au plus traité de façon anecdotique, dans la rubrique « insolites », plutôt que de figurer au-devant de l'actualité ». D'autant plus, ajoute-t-il, que «pour les Sénégalais tout ce qui est écrit et dit à la radio est vrai ». Bien qu'il faille relativiser cette toute puissance des médias depuis les études empiriques réalisées, dans les années 1940 par le Bureau of Social Applied Research (Université de Columbia), sous la houlette de Paul Lazarsfeld, il est toutefois difficile de nier la capacité qu'ont les médias de structurer les préoccupations du public en déterminant, par la sélection des évènements et des sujets, ce qui fera débat «conférant alors statuts, stéréotypes et images au réel» (Sfez, 1993, p. 1085). L'agenda setting (McCombs, Shaw, 1972) rend compte de cette fonction latente des médias d'information. Selon les tenants de cette théorie, ce rôle de sélection de l'information que les médias jouent en permanence, en direction du public, démontre que leur fonction n'est pas de dire aux gens ce qu'ils doivent penser, mais sur quoi ils doivent porter leur attention " puisqu'ils définissent le calendrier et la hiérarchie des évènements » (Rieffel, 2005, p. 25).

La théorie de l'agenda setting, centrée sur les effets cognitifs des médias plutôt que sur des changements d'attitude, d'opinions ou de comportements induits par les médias, est posée pour contredire la thèse des effets limités qui dominait alors la tradition empirique nord- américaine. L'inconvénient majeur de ces recherches «a été, probablement, qu'elles ont conduit trop de spécialistes à minimiser l'importance des mass media : sans doute, à trop vouloir insister sur l'influence personnelle et pas assez sur des influences extérieures, a-t-on été conduit à tomber dans l'excès inverse de celui qui avait précédé la publication de ces travaux» (Cayrol, 1991, p. 437). Le modèle de l'agenda setting, s'inscrivant pourtant dans la même tradition de recherches, c'est-à-dire celle des études sur les effets des médias, va tenter d'offrir une alternative en postulant que les médias ont un impact plus important qu'on veut bien le croire. Mais ne s'agit-il pas ici d'influence réciproque?

\section{Influence réciproque}

Si le Sénégal est cité comme modèle en matière de démocratie et de stabilité, il peine à décoller sur le plan économique. La grave crise économique qui secoue le monde et la majeure partie des pays d'Afrique subsaharienne ne l'a pas épargné. Un rapport du FMI a classé le Sénégal parmi les 25 pays les plus pauvres de la planète avec un PIB par habitant de 934,6 dollars en 2015 contre 1079 dollars en $2014^{16}$. Or, il est démontré que la crise économique offre un terrain fertile à toutes les formes d'obscurantisme. Les individus ont tendance à puiser dans leur tréfonds culturel, à avoir recours à l'irrationnel pour faire face à l'incertitude. Il n'est donc pas étonnant de constater que les pratiques mystico-religieuses traditionnelles qu'on croyait chassées par les religions du livre ${ }^{17}$ et la science moderne remontent aujourd'hui à la surface. 

déclarer qu'ils ne reconnaissent d'autres juridictions que celles de leurs pairs. Au Sénégal, par exemple, les professionnels sont en train de porter le combat pour la dépénalisation des délits de presse. Tant mieux si cela permet de reculer les limites de la liberté d'expression. Toutefois, liberté ne signifie pas licence, mais doit au contraire rimer avec sens de la responsabilité. Il est vrai que les lois sont muettes ou impuissantes par rapport à tout ce qui a trait à la voyance, à la divination, et au charlatanisme et/ou médecine traditionnelle. C'est encore vrai que ces phénomènes sont devenus un business lucratif pour les médias. Cela ne justifie nullement que les médias l'exploitent à outrance, quitte à sacrifier la santé de la population. Il est ici question d'éthique et d'intérêt national. Il n'est pas interdit aux médias de développer de temps en temps ce penchant naturel. Même dans les grandes démocraties, comme la France et les États-Unis, les médias en arrivent quelquefois à se faire les grands défenseurs de la patrie en péril. En France, cette tendance a été relevée dans un journal, et pas le moindre: le prestigieux quotidien Le Monde. Les réactions du Monde sur beaucoup de sujets sont principalement inspirées, à en croire Jean-Noël Jeanneney et Jacques Julliard, « par le souci de l'intérêt national » (Jeanneney, Julliard, p. 301).

\section{BIBLIOGRAPHIE}

Ouvrages et articles de revues 
Arous M. B. (dir.), Médias et conflits en Afrique, Paris, Karthala, Panos, 2001 Balle F., Introduction aux médias, Paris, PUF, 1999

Balle F., Les médias, Paris, Flammarion, 2000

Barry M., Histoire des médias au Sénégal. De la colonisation à nos jours, Paris, L'Harmattan, 2013

Bessette G., Communication et participation communautaire. Guide pratique de communication pour le développement, Québec, Presses de l'université de Laval, 2004

Cayrol R., Les médias. Presse écrite, radio, télévision, Paris, PUF, 1 ère édition, 1991

Damome E. et Delcroix F., « Risques naturels, santé et religion », in Pomel S. (dir.), Du risque en Afrique. Terrains et perspectives, Paris, Karthala, MSHA, 2015, p. 49-62

Derville G., Le pouvoir des médias. Mythes et réalités, Grenoble, PUG, 2005 Dieterlin G., Textes sacrés d'Afrique noire, Paris, Gallimard, 1965

FAO, La communication pour le développement, Manuel 1. Guide méthodologique d'élaboration d'une stratégie de communication multimédia, Rome 2002

Faye M., Presse privée écrite en Afrique francophone. Enjeux démocratiques. Paris, L'Harmattan, 2008

Faye M., «Bilan et perspectives de l'aide de l'état à la presse au Sénégal », Les Enjeux de l'information et de la communication, https://lesenjeux.univ-grenoble-alpes.fr/2015/02-Faye/ $n^{\circ} 16 / 1,2015$

Frère M.-S., Presse et démocratie en Afrique francophone. Les mots et les maux de la transition au Bénin et au Niger, Paris, Karthala, 2000

Frère M.-S. (dir.), Afrique centrale. Médias et conflits. Vecteurs de guerre ou acteurs de paix, Bruxelles, Coédition GRIP-Editions Complexe, 2005

Havard J.-F, « De la victoire du sopi à la tentation du nopi », in Politique Africaine $\mathrm{n}^{\circ}$ 96, décembre 2004, Paris, Karthala, p. 22-38

Hesseling G, Histoire politique du Sénégal, Paris, Karthala, 1985

Hester A. L., To W. L. J., Journalisme et tiers monde, Bruxelles, De Boeck-Wesmael, 1989

Jeanneney J.-N., Julliard J., Le Monde de Beuve-Merry ou le métier d'Alceste, Paris, Editions du Seuil, 1979

Karikari K., «Présentation : 50 ans depuis l'indépendance du Ghana », in Barratt E. et Berger G. (éd.), 2007, 50 ans de journalisme. Les médias africains depuis l'indépendance du Ghana,

The African Ediror Forum, Highway Africa, 2007

Lenoble-Bart A. et Tudesq A.-J. (dir.), Pour connaître les médias d'Afrique subsaharienne. Problématiques, sources et ressources, IFAS-IFRA-MSHA-Karthala, 2008

Loum, N., Les médias et l'État au Sénégal. L'impossible autonomie, L'Harmattan, 2003

McCombs M., Shaw D., « The agenda-setting function of mass-média », Public Opinion Quarterly, $\mathrm{n}^{\circ} 36,1972$, p. 176-187

Monteil V., L'islam noir. Une religion à la conquête de l'Afrique, Paris, Editions du Seuil, 1980

Pasquier R., «Les débuts de la presse au Sénégal », Cahier d'études africaines, I X, n 33, Paris :

Éditions de l'EHESS, 1962, p. 477-490 
Paye M., « La presse et le pouvoir », in Diop M.C., Sénégal. Trajectoires d'un État, Paris- Dakar, Karthala, 1992, p. 331-377

Rieffel R., Sociologie des médias, Paris, Ellipses, $2^{\mathrm{e}}$ édition enrichie et actualisée, 2005 Sfez L., Dictionnaire critique de la communication, Paris, PUF, 1993, Tome 2 Tchakhotine S., Le viol des foules par la propagande politique, Gallimard, 1952 Thomas L.-V, Luneau R., La terre africaine et ses religions, L'Harmattan, 1995

Touré B., « Presse et pluralisme au Sénégal », in Presse francophoned'Afrique : vers le pluralisme, Actes du colloque Panos/Ujao/Unesco, L'Harmattan, 1991 Tudesq A.-J., Les médias en Afrique, Paris, Ellipses, 1999 Tudesq A-J, Feuilles d'Afrique, Talence, MSHA, 1995

Articles, thèses, mémoires

Dia S., « De la TSF coloniale à l'ORTS : évolution de la place et du rôle de la radiodiffusion au Sénégal(1911-1986) », Thèsede $3^{\mathrm{e}}$ cycle en sciences de l'information et de la Communication, Université de Bordeaux 3, 1987

Ndiaye M., «Le rôle des médias dans la réalisation de l'alternance politique au Sénégal », Mémoire de DUR en sciences de l'information et de la communication, Université de Bordeaux 3 , 2001-2002

Tedesco M., « Communication pour le développement et radios communautaires : Cas du Népal », Mémoire de Master en Coopération internationale. Action humanitaire et politique de développement, Université Paris I Panthéon Sorbonne, 2007-2008.

\section{NOTES}

1. Mansary A. L., « Nouvelle profanation de cimetière : A Pikine, les morts n'ont pas le repos éternel », Le quotidien, 5 décembre 2015.

2. Kane D. « Neuf mois après son investiture, le Président de la République déménage enfin au Palais ", Le Quotidien, 20 décembre 2012. Nettali, le quotidien exclusivement sur le net, dans : « Macky déménage au palais », va plus loin : «Les détracteurs du chef de l'État se plaisaient même à dire que s'il n'a toujours pas passé la nuit au palais, c'est parce que les saltigués (devins) déminaient le palais pour le dépouiller des gris-gris et eaux bénites qui y seraient enfouis ou versés ", 24 décembre 2012.

3. L'ex-président Abdoulaye Wade a ouvertement accusé son ex-allié Pape Diop, ancien président du Sénat, d'avoir sacrifié un albinos lors d'une réunion du comité directeur de son parti. Une sortie qui a créé un tollé au Sénégal.

4. http://www.buzzsenegal.com/Saviezvous/mais-jusqu-rsquo-ou-ira-iran-ndao-parkh_n_843.html, consulté le 15 novembre 2015.

5. Stades, 30 janvier 2016.

6. Mot wolof qui signifie littéralement « bien être».

7. «Reug Reug ».

8. «L'Ordre des médecins traîne le tradi- praticien de la SenTv, Samba Ndiaye, à la barre », IGFM, 21 novembre 2014, consulté le 28 janvier 2016, http://www.igfm.sn/ lordre-des-medecins-traine-le-tradi-praticien-de-la- sentv-samba-ndiaye-a-la-barre.

9. http://www.cnra.sn/do/n201404, janvier-2014/

10. http://www.cnra.sn/do/avis-trimestriel-janvier-fevrier-mars-2014/

11. http://www.cnra.sn/do/avis-trimestriel-juillet-aout-septembre-2014 
12. Mbengue $C$. T., "Sénégal: Publicités mensongères des charlatans - Les médias au banc des accuses", Sud quotidien, 4 octobre 2015.

13. Dossier sur la violence au Sénégal : un marché lucratif, La Gazette, 18 février 2014. http://www.lagazette.sn/dossier-sur-la-voyance-au-senegal-un-marche-lucratif consulté le 4 décembre 2015.

14. Ibidem.

15. Ibidem.

16. Seneweb.com, Le 25 octobre 2015, http://www.seneweb.com/news/Economie/le-senegalclasse-parmi-les-25-pays-les-_n_166797.html.

17. Ces religions se sont posées en s'opposant radicalement et violemment aux cultes et pratiques traditionnels, dits «païens» et « idolâtres ».

18. Dossier sur la violence au Sénégal : un marché lucratif, La Gazette, 18 février 2014. http:// www.lagazette.sn/dossier-sur-la-voyance-au-senegal-un-marche-lucratif consulté le 4 décembre 2015.

19. Combien rapportent les serveurs (?) : Silence radio sur la bande Fm, sauf chez Ndella Madior, Ferloo, 19 août 2013.

\section{RÉSUMÉS}

Le recours aux pratiques mystiques, à la voyance et/ou divination, à la médecine traditionnelle est devenu tellement manifeste dans notre société actuelle qu'il n'est pas péremptoire de décréter le retour du traditionalisme. Les charlatans, voyants, guérisseurs, etc., occupent de plus en plus l'espace et fonctionnent comme soupape de sécurité pour une population en détresse. Ils exploitent la misère matérielle et/ou morale de la masse en lui garantissant des solutions à tous ses maux. Pour s'en rendre compte, il suffit de suivre l'actualité de leurs pratiques à travers les émissions consacrées à la voyance, aux féticheurs et guérisseurs qui pullulent aujourd'hui sur les fréquences radios et chaînes de télévision.

Au début simple phénomène de presse écrite, le phénomène de la voyance, de la divination et du charlatanisme s'est maintenant généralisé à tous les médias. En leur offrant cette visibilité, les médias font ainsi la publicité et donnent de la légitimité à ces nouveaux acteurs qui semblent pouvoir trouver remède à tout. Les médias, ce faisant, ont-ils conscience de desservir la médecine moderne et de fragiliser la santé physique et mentale du public? Ou le font-ils en toute connaissance de cause et tout simplement pour répondre aux attentes des auditoires friands de ces dites informations? Cette étude analyse ce regain d'intérêt des médias pour ces pratiques traditionalistes et les risques pour le développement.

The recourse to mystical practices, foretelling, divination, and traditional medicine are significant in our society, which are not conclusive to claim a return to traditionalism. Quakers, seers, healers, etc., are more and more used by anxious people. They manage their poverty and moral anxiousness by guaranteeing solutions to all ills. As evidence, just listen to the news of their practices through radios and televisions broadcasts.

As simple news papers event at the beginning, foreseeing and quackery have been extended to all media. By providing visibility to these practices, the media give legitimacy to these new actors who seem to find cure for all kind of diseases. In doing so, do the media have any consciousness 
to deserve modern medicine, while undermining the physical and mental health to the public? Or do they act in purpose, just to meet the expectations of audiences who are fond of this kind of information. This study is an analysis of the new media care for those traditional practices with the risks linked to the development.

L'utilizzo di pratiche mistiche, la veggenza e / o la divinazione, la medicina tradizionale sono diventate così evidenti nella nostra società che non è lontano il ritorno del tradizionalismo.

Ciarlatani, veggenti, guaritori, ecc, occupano sempre più spazio e riconinciano a funzionare come valvola di sicurezza per la popolazione in difficoltà. Sfruttano la povertà materiale e/o morale garantendo soluzioni per tutti i mali. Basta semplicemente seguire le notizie delle loro pratiche attraverso le trasmissioni sulla veggenza, stregoni e guaritori che ora pullulano sulle frequenze radio e canali televisivi. All inizio era un semplice fenomeno di stampa, il fenomeno di veggenza, la divinazione e ciarlataneria sono ora estesi a tutti i media.

Offrendo loro questa visibilità, i media, fanno pubblicita e danno legittimità ai nuovi attori che sembrano trovare la cura per tutto. I mezzi di comunicazione, così facendo, hanno essi la coscienza di nuocere alla medicina moderna minando la salute fisica e mentale del pubblico ? o lo fanno consapevolmente e solo per soddisfare le aspettative del pubblico appassionato di questo genere di informazioni. Questo studio analizza l' interesse dei media per queste pratiche tradizionaliste e i rischi per lo sviluppo.

\section{INDEX}

Parole chiave : media, informazione, comunicazione, sviluppo, comunicazione per lo sviluppo, la salute, la chiaroveggenza, la divinazione, la medicina tradizionale.

Keywords : media, information, communication, development, communication for development, health, clairvoyance, divination, traditional medicine.

Mots-clés : médias, information, communication, développement, communication pour le développement, santé, voyance, divination, médecine traditionnelle.

\section{AUTEUR}

\section{SEYDOU NOUROU SALL}

Université Gaston Berger/ Saint-Louis, Sénégal 